\title{
Un théorème de finitude
}

\author{
( A Theorem of Finitude)
}

\begin{abstract}
ALEXANDRU IOAN BADULESCU with an appendix by PAUL BROUSSOUS

Université de Poitiers, UFR Sciences SP2MI, Département de mathématiques, UMR CNRS 6086 Groupes de Lie et géométrie, Téléport 2, Boulevard Marie et Pierre Curie, BP 30179, 86962 Futuroscope Chasseneuil Cedex, France.e-mail: badulesc,broussous@, wallis.sp2mi.univ-poitiers. fr
\end{abstract}

(Received: 7 August 2000; accepted in final form: 7 May 2001)

\begin{abstract}
We prove that, for an inner form of $G L_{n}$, the number of classes of cuspidal automorphic representations with fixed central character and fixed factor at almost every place is finite. We also prove, in the local situation, relations between the level and the $\epsilon$-factor of an irreducible smooth representation.
\end{abstract}

Mathematics Subject Classifications (2000). 20G25, 20G05.

Key words. level, $\epsilon$-factor, automorphic representation.

\section{Introduction}

Soit $F$ un corps global et soit $A$ une algèbre centrale simple de dimension finie sur $F$. Soient $F^{*}(\mathbb{A})$ et $A^{*}(\mathbb{A})$ les groupes des adèles de $F^{*}$ et $A^{*}$ respectivement. On identifie $F^{*}$ au centre de $A^{*}$ et $F^{*}(\mathbb{A})$ au centre de $A^{*}(\mathbb{A})$. Pour toute place $v$ de $F$ on note $F_{v}$ et $A_{v}$ les localisés de $F$ et de $A$ en $v$. Le but principal de cet article est d'établir le résultat suivant, au moins quand la caractristique de $F$ est nulle:

THÉORÈME 1.1. Soit $V$ un ensemble fini de places finies de F. Si pour toute place $v \notin V$ on fixe une représentation lisse irréductible $\pi_{v}$ de $A_{v}^{*}$, alors il existe au plus un nombre fini de classes d'équivalence de représentations automorphes cuspidales $\pi$ de $A^{*}(\mathbb{A})$ telles que, pour tout $v \notin V$, la composante locale de $\pi$ à la place $v$ soit équivalente $\grave{a} \pi_{v}$.

La démonstration se base sur le résultat de nature locale suivant: Soit $F$ un corps local non archimédien, soit $D$ une algèbre à division centrale de dimension finie $d^{2}$ sur $F$, soit $r$ un entier strictement positif et posons $A=M_{r}(D)$. On pose $n=r d$. Soit $\mathcal{O}$ l'anneau des entiers de $D$ et soit $P$ son idéal maximal. Si $\pi$ est une représentation lisse irréductible de $A^{*}$, on définit le niveau de $\pi$ (notation niv $(\pi)$ ) comme étant un entier égal à 0 si $\pi$ a un vecteur fixe non nul sous $G L_{r}(\mathcal{O})$ et égal au plus petit entier $k$ tel que $\pi$ ait un vecteur non nul fixe sous $1+M_{r}\left(P^{k}\right)$ sinon. On fixe un caractère 
additif non trivial $\psi$ de $F$. Si $\pi$ est une représentation lisse irréductible de $A^{*}$, soit $\epsilon(\pi ; s)$ le facteur de Godement-Jacquet associé à $\pi$ et $\psi$. D'après [GJ], on a

$$
\epsilon(\pi ; s)=C(\pi) q^{(n a(\psi)-m(\pi)) s}
$$

où $C(\pi)$ est une constante complexe non nulle, $a(\psi)$ est un certain entier qui ne dépend que de $\psi$ et $m(\pi)$ est un entier qui ne dépend que de $\pi$ (et pas de $\psi$ ) appelé ici le conducteur de $\pi$. Avec ces notations on a:

THÉORÈME 1.2. (a) Si $\pi$ est une représentation lisse irréductible cuspidale de $A^{*}$, alors on a

$$
\operatorname{niv}(\pi) \leqslant \frac{m(\pi)}{r}-d+2 .
$$

(b) Si $\pi$ est une représentation lisse irréductible quelconque de $A^{*}$, alors on a

$$
\operatorname{niv}(\pi) \leqslant m(\pi)-n+2 r .
$$

Le théorème 1.1 est d'une grande utilité pour établir une démonstration limpide de la correspondance de Jacquet-Langlands locale (et probablement globale) entre $G L_{n}$ et ses formes intérieures. Rogawski d'ailleurs, pour les besoins de sa démonstration de la correspondance de Jacquet-Langlands entre $G L_{n}$ et une algèbre à division ([Ro]) a prouvé un résultat analogue dans le cas particulier ou $A$ est une algèbre à division telle que pour toutes les places $v$ de $F, A_{v}$ est ou bien une algèbre de matrices sur $F_{v}$ ou bien une algèbre à division. Dans sa démonstration, Rogawski utilise la relation entre le niveau et le conducteur d'une représentation, bien connue quand le groupe est le groupe des éléments inversibles d'une algèbre à division. C'est par la même méthode que nous obtiendrons ici le théorème 1.1 à partir du théorème 1.2.

Je remercie tout spécialement Guy Henniart qui est à l'origine de cet article ; c'est lui qui m'a signalé le fait que les derniers travaux sur les types devraient permettre maintenant d'obtenir des relations entre le niveau et le conducteur, susceptibles d'impliquer le théorème 1.1. Je remercie aussi Paul Broussous d'avoir écrit un appendice au présent article, où il montre un résultat qui est crucial pour mes démonstrations.

\section{Le théorème 1.2(a) implique le théorème 1.2(b)}

Nous montrerons dans cette section que le théorème 1.2(a) implique le théorème 1.2(b), et cela sans restriction sur la caractéristique du corps de base.

Soit $F$ un corps local non archimédien et soit $A$ une algèbre centrale simple de dimension finie sur $F$. Il existe alors une algèbre à division $D$ centrale sur $F$ et un entier strictement positif $r$ tel que $A$ soit isomorphe à $M_{r}(D)$. Par la suite, on identifiera $A$ à $M_{r}(D)$ et le groupe des éléments inversibles de $A$, qu'on note $G$, à $G L_{r}(D)$. La dimension de $D$ sur $F$ est le carré d'un nombre entier strictement positif $d$ : $\operatorname{dim}_{F} D=d^{2}$. Posons $r d=n$. Soient $\mathcal{O}_{F}$ l'anneau des entiers de $F$ et $P_{F}$ son idéal 
maximal. On fixe une uniformisante $\pi_{F}$ de $F$. Soient $\mathcal{O}_{D}$ l'anneau des entiers de $D$ et $P_{D}$ son idéal maximal. On fixe une uniformisante $\pi_{D}$ de $D$ telle que $\pi_{D}^{d}=\pi_{F}$.

On fixe par convention une paire parabolique minimale standard $\left(A_{0} ; P_{0}\right)$ où $A_{0}$ est le tore diagonal et $P_{0}$ est le groupe des matrices triangulaires supérieures. On ne considérera que des sous-groupes paraboliques standard et des sous-groupes de Levi standard. Ces derniers s'écrivent donc comme un produit diagonal de blocs $B_{1} \times B_{2} \times \cdots \times B_{k}$ où chaque $B_{i}$ est isomorphe à un $G L_{r_{i}}(D)$ et $\sum r_{i}=r$. Soit $K^{0}$ égal à $G L_{r}\left(\mathcal{O}_{D}\right)$ (sous-groupe compact maximal de $G$ ). On pose, pour tout $l \in \mathbb{N}^{*}$, $K^{l}=1+M_{n}\left(P_{D}^{l}\right)$. Les sous-groupes $K^{l}$ forment une base de voisinages ouverts et compacts de l'élément neutre. Ces choix vérifient pour $l>0$ les conditions posées dans [Be], 2.1(b), à savoir $A_{0}$ est un tore déployé maximal de $G, K^{l}$ vérifie: pour tout sous-groupe parabolique $P$ de $G$ en position standard par rapport à la paire $\left(A_{0} ; P_{0}\right)$, si $P=L U$ est la décomposition de Levi standard de $P$ et $P=L \bar{U}$ est la décomposition de Levi standard du parabolique opposé, alors on a: $K^{l}=\left(U \cap K^{l}\right)$ $\left(L \cap K^{l}\right)\left(\bar{U} \cap K^{l}\right)$.

Si $\pi$ est une représentation lisse irréductible de $G$, on appelle niveau de $\pi$ le plus petit entier positif $l$ tel que $\pi$ ait un vecteur non nul fixe sous $K^{l}$. On note cet entier $\operatorname{niv}(\pi)$.

Fixons une fois pour toutes dans cette section un caractère additif non trivial $\psi$ de $F$.

Soit $\pi$ une représentation admissible de $G$. Dans [GJ] on attache à $\pi$ les fonctions

$$
L(s ; \pi), \quad \epsilon(s ; \pi ; \psi) \quad \text { et } \quad \epsilon^{\prime}(s ; \pi ; \psi)=\epsilon(s ; \pi ; \psi) L(1-s ; \check{\pi}) L(s ; \pi)^{-1}
$$

où $\check{\pi}$ est la représentation contragradiente de $\pi$. Soit $a(\psi)$ le plus petit entier qui vérifie $\operatorname{ker} \psi \supseteq P_{F}^{a(\psi)}$. On a alors

$$
\epsilon(s ; \pi ; \psi)=C(\pi) q^{(n a(\psi)-m(\pi)) s}
$$

où $C(\pi)$ est une constante complexe non nulle et $m(\pi)$ est un entier qui ne dépend que de $\pi$ et pas de $\psi$ (et dont on ne sait pas a priori s'il est positif ou pas). On appelle $m(\pi)$ le conducteur de $\pi$.

Remarque. Ces considérations se trouvent, par exemple, dans [GJ], page 33. La valeur $n a(\psi)$ qui apparaît dans la relation (1) peut être retrouvée par la formule (3.3.5) de [GJ]. Cette valeur sera nulle si l'on choisit un caractère $\psi$ nonramifié, i.e. trivial sur $\mathcal{O}_{F}$ et non trivial sur $P_{F}^{-1}$. Elle se simplifiera de toutes façons dans les calculs effectifs faits par la suite. Nous aurions donc pu choisir un caractère non ramifié pour simplifier ces calculs. Le choix (encombrant) d'un caractère $\psi$ éventuellement ramifié, comme plus haut, vient de ce qu'on utilisera un résultat de Bushnell et Fröhlich qui est énoncé pour un caractère particulier. Néanmoins, remarquons que, malgré ce passage par un résultat dépendant de $\psi$, les résultats du théorème 1.2 en sont indépendants. 
PROPOSITION 2.1. Supposons que 1.2(a) soit vrai pour toute algèbre A comme plus haut. Alors 1.2(b) est vrai.

Démonstration. Supposons que 1.2(a) soit vérifié. Soit $\pi$ une représentation lisse irréductible de $G$. Si $\pi$ est une représentation cuspidale, le résultat est impliqué par 1.2(a). Supposons maintenant que $\pi$ ne soit pas cuspidale. Soient $P$ un sous-groupe parabolique standard de $G, L$ son sous-groupe de Levi standard et $\rho$ une représentation cuspidale de $L$, tels que $\pi$ soit un quotient de $\operatorname{ind}_{P}^{G} \rho$. Écrivons $L=B_{1} \times B_{2} \times \cdots \times B_{l}$ où, pour tout

$$
i \in\{1,2 \ldots, l\}, \quad B_{i} \simeq G L_{r_{i}}(D) \quad \text { et } \quad \rho=\rho_{1} \otimes \rho_{2} \otimes \cdots \otimes \rho_{l},
$$

où, pour tout $i \in\{1,2 \ldots l\}, \rho_{i}$ est une représentation cuspidale de $B_{i}$.

LEMME 2.2. Avec les notations déjà fixées, on a $m(\pi) \geqslant \sum_{i=1}^{l} m\left(\rho_{i}\right)$.

Démonstration. Par la proposition 3.5 et le corollaire 3.6 de [GJ] on a

$$
\epsilon^{\prime}(s, \pi ; \psi)=\prod_{i=1}^{l} \epsilon^{\prime}\left(s, \rho_{i} ; \psi\right)
$$

On revient à la définition de la fonction $\epsilon^{\prime}$ et on remplace:

$$
\epsilon(s ; \pi ; \psi) L(1-s ; \check{\pi}) L(s ; \pi)^{-1}=\prod_{i=1}^{l} \epsilon\left(s ; \rho_{i} ; \psi\right) \prod_{i=1}^{l} L\left(1-s ; \check{\rho}_{i}\right) \prod_{i=1}^{l} L\left(s ; \rho_{i}\right)^{-1} .
$$

Nous savons par la proposition 3.5 et le corollaire 3.6 de [GJ] que

$$
\frac{L(s ; \pi)}{\prod_{i=1}^{l} L\left(s ; \rho_{i}\right)}=R\left(q^{-s}\right)
$$

et

$$
\frac{L(1-s ; \check{\pi})}{\prod_{i=1}^{l} L\left(1-s ; \check{\rho}_{i}\right)}=R^{\prime}\left(q^{s-1}\right)
$$

où $R$ et $R^{\prime}$ sont des polynômes non nuls à coefficients complexes. Donc

$$
\epsilon(s ; \pi ; \psi) R^{\prime}\left(q^{s-1}\right)=\left(\prod_{i=1}^{l} \epsilon\left(s ; \rho_{i} ; \psi\right)\right) R\left(q^{-s}\right)
$$

ou, encore, si deg $R^{\prime}$ est le degré de $R^{\prime}$ :

$$
\epsilon(s ; \pi ; \psi) q^{-\left(\operatorname{deg} R^{\prime}\right) s} R^{\prime}\left(q^{s-1}\right)=\left(\prod_{i=1}^{l} \epsilon\left(s ; \rho_{i} ; \psi\right)\right) q^{-\left(\operatorname{deg} R^{\prime}\right) s} R\left(q^{-s}\right) .
$$


Maintenant, $q^{-\left(\operatorname{deg} R^{\prime}\right) s} R^{\prime}\left(q^{s-1}\right)=R^{\prime \prime}\left(q^{-s}\right)$ où $R^{\prime \prime}$ est un polynôme de degré au plus $\operatorname{deg} R^{\prime}$; il y a en fait égalité $\operatorname{deg} R^{\prime \prime}=\operatorname{deg} R^{\prime}$ parce que $R^{\prime}(0) \neq 0$. On a donc

$$
C(\pi) q^{(n a(\psi)-m(\pi)) s} R^{\prime \prime}\left(q^{-s}\right)=\left(\prod_{i=1}^{l} C\left(\rho_{i}\right) q^{\left(r_{i} d a(\psi)-m\left(\rho_{i}\right)\right) s}\right) q^{-\left(\operatorname{deg} R^{\prime}\right) s} R\left(q^{-s}\right) .
$$

Comme

$$
n a(\psi)=\sum_{i=1}^{l} r_{i} d a(\psi)
$$

l'expression précédente se simplifie. Ensuite, en multipliant, s'il le faut, les deux termes de l'égalité par la même puissance de $q^{-s}$ afin d'obtenir une égalité de polynômes en $q^{-s}$ (c'est parce qu'a priori nous ne connaissons pas le signe des $m(\pi)$ ), par l'égalité des degrés de ces deux polynômes on trouve

$$
m(\pi)+\operatorname{deg} R^{\prime \prime}=\sum_{i=1}^{l} m\left(\rho_{i}\right)+\operatorname{deg} R^{\prime}+\operatorname{deg} R .
$$

En tenant compte de $\operatorname{deg} R^{\prime \prime}=\operatorname{deg} R^{\prime}$ on a finalement $m(\pi) \geqslant \sum_{i=1}^{l} m\left(\rho_{i}\right)$.

Passons maintenant à la démonstration de la proposition 2.1. On applique le lemme 2.2 et ensuite on applique la proposition 1.2(a) aux représentations cuspidales $\rho_{i}$ :

$$
m(\pi) \geqslant \sum_{i=1}^{l} m\left(\rho_{i}\right) \geqslant \sum_{i=1}^{l}\left(r_{i} \operatorname{niv}\left(\rho_{i}\right)+d r_{i}-2 r_{i}\right)=n-2 r+\sum_{i=1}^{l} r_{i} \operatorname{niv}\left(\rho_{i}\right) .
$$

Maintenant, de la proposition 3.5.2 [Be], en tenant compte que nos choix vérifient l'hypothèse de cette proposition, le niveau de $\pi$ est inférieur ou égal au maximum des niveaux des $\rho_{i}$. Comme le maximum des niveaux des représentations $\rho_{i}$ est inférieur à la somme $\sum_{i=1}^{l} r_{i} \operatorname{niv}\left(\rho_{i}\right)$, on obtient le résultat.

\section{Le théorème 1.2(b) implique le théorème 1.1}

Soit $F$ un corps global et soit $A$ une algèbre centrale simple de dimension finie sur $F$. Soient $F^{*}(\mathbb{A})$ et $A^{*}(\mathbb{A})$ les groupes des adèles de $F^{*}$ et $A^{*}$ respectivement. On identifie $F^{*}$ au centre de $A^{*}$ et $F^{*}(\mathbb{A})$ au centre de $A^{*}(\mathbb{A})$. Pour toute place $v$ de $F$ on note $F_{v}$ et $A_{v}$ les localisés de $F$ et de $A$ en $v$. Soit $V$ un ensemble fini de places finies de $F$. Pour toute place $v \notin V$ on fixe une représentation lisse irréductible $\pi_{v}$ de $A_{v}^{*}$.

PROPOSITION 3.1. Soit X l'ensemble des classes d'équivalence de représentations automorphes cuspidales irréductibles $\pi$ de $A^{*}(\mathbb{A})$ telles que, pour tout $v \notin V$, la composante locale de $\pi$ à la place $v$ est équivalente à $\pi_{v}$. Supposons que le résultat $1.2(\mathrm{~b})$ soit vrai. Alors $X$ est fini.

Démonstration. Supposons que $X$ ne soit pas vide et soit $\tilde{\pi} \in X$. Fixons cette représentation une fois pour toutes. Soit maintenant $\tilde{\pi}^{\prime} \in X$. On va évaluer 
$\prod_{v \in V} \epsilon\left(s ; \tilde{\pi}_{v}^{\prime} ; \psi_{v}\right)$. Grâce aux travaux de [GJ] on sait que $\prod_{v \notin V} L\left(s ; \tilde{\pi}_{v}^{\prime}\right)$ converge absolument pour $\operatorname{Re}(s)$ assez grand et que la fonction limite se prolonge en une fonction $L_{\mathbb{K}, \tilde{\pi}^{\prime}}$ méromorphe dans tout le plan. Des résultats analogues valent pour

$$
\prod_{v \notin V} L\left(1-s ; \check{\tilde{\pi}}_{v}^{\prime}\right) \quad \text { et } \prod_{v \notin V} \epsilon\left(s ; \tilde{\pi}_{v}^{\prime} ; \psi_{v}\right) .
$$

Nous notons de la même manière $L_{K, \tilde{\tilde{\pi}}^{\prime}}$ et $\epsilon_{\mathcal{K}, \tilde{\pi}^{\prime}}$ les fonctions méromorphes ainsi obtenues. Ces considérations sont valables pour $\tilde{\pi}$ également et nous adoptons des notations analogues.

En vertu de l'équation fonctionnelle globale ([GJ], 13.8), on a l'égalité suivante

$$
\begin{aligned}
& \prod_{v \in V} \epsilon\left(s ; \tilde{\pi}_{v}^{\prime} ; \psi_{v}\right) \prod_{v \in V} L\left(1-s ; \check{\tilde{\pi}}_{v}^{\prime}\right) \prod_{v \in V} L\left(s ; \tilde{\pi}_{v}^{\prime}\right)^{-1} \epsilon_{\mathbb{K}, \tilde{\pi}^{\prime}}(s) L_{K, \tilde{\pi}^{\prime}}(s) L_{K, \tilde{\pi}^{\prime}}^{-1}(s) \\
& =\prod_{v \in V} \epsilon\left(s ; \tilde{\pi}_{v} ; \psi_{v}\right) \prod_{v \in V} L\left(1-s ; \check{\tilde{\pi}}_{v}\right) \prod_{v \in V} L\left(s ; \tilde{\pi}_{v}\right)^{-1} \epsilon_{\mathcal{X}, \tilde{\pi}}(s) L_{K, \tilde{\tilde{\pi}}}(s) L_{\alpha, \tilde{\pi}}^{-1}(s) .
\end{aligned}
$$

Aux places ne se trouvant pas dans $V$, les composantes locales des deux représentations automorphes $\tilde{\pi}^{\prime}$ et $\tilde{\pi}$ sont équivalentes. Nous avons donc $\epsilon_{\bar{R}, \tilde{\pi}^{\prime}}=\epsilon_{\mathbb{K}, \tilde{\pi}, \tilde{r}}$, $L_{K, \tilde{\tilde{\pi}^{\prime}}}=L_{K, \tilde{\tilde{\pi}}}$ et $L_{\mathbb{K}, \tilde{\pi}^{\prime}}=L_{\mathbb{K}, \tilde{\pi}^{\prime}}$. On peut donc simplifier l'égalité plus haut et la ramener à:

$$
\begin{aligned}
& \prod_{v \in V} \epsilon\left(s ; \tilde{\pi}_{v}^{\prime} ; \psi_{v}\right) \prod_{v \in V} L\left(1-s ; \check{\tilde{\pi}}_{v}^{\prime}\right) \prod_{v \in V} L\left(s ; \tilde{\pi}_{v}^{\prime}\right)^{-1} \\
& \quad=\prod_{v \in V} \epsilon\left(s ; \tilde{\pi}_{v} ; \psi_{v}\right) \prod_{v \in V} L\left(1-s ; \check{\tilde{\pi}}_{v}\right) \prod_{v \in V} L\left(s ; \tilde{\pi}_{v}\right)^{-1} .
\end{aligned}
$$

Posons, pour toute place $v \in V$,

$$
\begin{aligned}
& L\left(s ; \tilde{\pi}_{v}\right)=P_{v}\left(q_{v}^{-s}\right)^{-1}, L\left(s ; \tilde{\pi}_{v}^{\prime}\right)=P_{v}^{\prime}\left(q_{v}^{-s}\right)^{-1}, \\
& L\left(s ; \check{\tilde{\pi}}_{v}\right)=\check{P}_{v}\left(q_{v}^{-s}\right)^{-1}, L\left(s ; \check{\tilde{\pi}}_{v}^{\prime}\right)=\check{P}_{v}^{\prime}\left(q_{v}^{-s}\right)^{-1},
\end{aligned}
$$

où $P_{v}, P_{v}^{\prime}, \check{P}_{v}, \check{P}_{v}^{\prime}$ sont des polynômes à coefficients complexes. Nous avons donc:

$$
\prod_{v \in V} \epsilon\left(s ; \tilde{\pi}_{v}^{\prime} ; \psi_{v}\right) \check{P}_{v}^{\prime}\left(q_{v}^{s-1}\right)^{-1} P_{v}^{\prime}\left(q_{v}^{-s}\right)=\prod_{v \in V} \epsilon\left(s ; \tilde{\pi}_{v} ; \psi_{v}\right) \check{P}_{v}\left(q_{v}^{s-1}\right)^{-1} P_{v}\left(q_{v}^{-s}\right) .
$$

Comme nous l'avons déjà fait auparavant, on pose

$$
\check{P}_{v}^{\prime}\left(q_{v}^{s-1}\right)=q_{v}^{\left(\operatorname{deg} \check{P}_{v}^{\prime}\right) s} S_{v}^{\prime}\left(q_{v}^{-s}\right) \quad \text { et } \quad \check{P}_{v}\left(q_{v}^{s-1}\right)=q_{v}^{\left(\operatorname{deg} \check{P}_{v}\right) s} S_{v}\left(q_{v}^{-s}\right)
$$

où $S_{v}^{\prime}$ est un polynôme de même degré que $\check{P}_{v}^{\prime}$ et $S_{v}$ est un polynôme de même degré que $\check{P}_{v}$. Remplaçons:

$$
\begin{aligned}
& \prod_{v \in V} \epsilon\left(s ; \tilde{\pi}_{v}^{\prime} ; \psi_{v}\right) q_{v}^{-\left(\operatorname{deg} \check{\check{P}}_{v}^{\prime}\right) s} P_{v}^{\prime}\left(q_{v}^{-s}\right) S_{v}\left(q_{v}^{-s}\right) \\
& \quad=\prod_{v \in V} \epsilon\left(s ; \tilde{\pi}_{v} ; \psi_{v}\right) q_{v}^{-\left(\operatorname{deg} \check{P}_{v}\right) s} P_{v}\left(q_{v}^{-s}\right) S_{v}^{\prime}\left(q_{v}^{-s}\right) .
\end{aligned}
$$


Posons, pour tout $v \in V$,

$$
\epsilon\left(s ; \tilde{\pi}_{v} ; \psi_{v}\right)=a_{v} q_{v}^{\left(n a\left(\psi_{v}\right)-m\left(\tilde{\pi}_{v}\right)\right) s}
$$

où $a_{v}$ est une constante complexe non nulle et

$$
\epsilon\left(s ; \tilde{\pi}_{v}^{\prime} ; \psi_{v}\right)=a_{v}^{\prime} q_{v}^{\left(n a\left(\psi_{v}\right)-m\left(\tilde{\pi}_{v}^{\prime}\right)\right) s}
$$

où $a_{v}^{\prime}$ est une constante complexe non nulle. On peut supposer que les $q_{v}$ sont tous égaux, sans quoi on les sépare. $\mathrm{Si}$ on remplace dans (2), on simplifie par $q^{\left(n \Sigma_{v \in V} a\left(\psi_{v}\right)\right) s}$ et on multiplie éventuellement les deux membres avec une puissance de $q^{-s}$ afin d'obtenir une égalité de polynômes en $q^{-s}$, en comparant le degré des deux polynômes qui sont égaux pour une infinité de valeurs complexes, on obtient:

$$
\begin{aligned}
& \sum_{v \in V} m\left(\tilde{\pi}_{v}^{\prime}\right)+\sum_{v \in V} \operatorname{deg} \check{P}_{v}^{\prime}+\sum_{v \in V} \operatorname{deg} P_{v}^{\prime}+\sum_{v \in V} \operatorname{deg} S_{v} \\
& =\sum_{v \in V} m\left(\tilde{\pi}_{v}\right)+\sum_{v \in V} \operatorname{deg} \check{P}_{v}+\sum_{v \in V} \operatorname{deg} P_{v}+\sum_{v \in V} \operatorname{deg} S_{v}^{\prime}
\end{aligned}
$$

soit

$$
\sum_{v \in V} m\left(\tilde{\pi}_{v}^{\prime}\right)=\sum_{v \in V} m\left(\tilde{\pi}_{v}\right)+\sum_{v \in V} \operatorname{deg} P_{v}-\sum_{v \in V} \operatorname{deg} P_{v}^{\prime}
$$

puisque $\operatorname{deg} S_{v}^{\prime}=\operatorname{deg} \check{P}_{v}^{\prime}$ et $\operatorname{deg} S_{v}=\operatorname{deg} \check{P}_{v}$. Finalement on trouve

$$
\sum_{v \in V} m\left(\tilde{\pi}_{v}^{\prime}\right) \leqslant \sum_{v \in V} m\left(\tilde{\pi}_{v}\right)+\sum_{v \in V} \operatorname{deg} P_{v}
$$

ce qui implique (rappelons que $\tilde{\pi} \in X$ a été fixée) que, si $X$ est non vide, alors il existe une constante réelle $c$ telle que pour toute $\tilde{\pi}^{\prime} \in X$ on ait

$$
\sum_{v \in V} m\left(\tilde{\pi}_{v}^{\prime}\right) \leqslant c
$$

Nous reprenons les notations 'locales' des sections précédentes: pour toute place finie $v$ de $F, A_{v}$ est isomorphe à une algèbre de matrices $M_{r_{v}}\left(D_{v}\right)$ où $D_{v}$ est une algèbre à division centrale de dimension $d_{v}^{2}$ sur $F_{v}$, et on note $\mathcal{O}_{D_{v}}$ l'anneau des entiers de $D_{v}$ et $P_{D_{v}}$ l'idéal maximal de $\mathcal{O}_{D_{v}}$. On pose $K_{v}^{0}=G L_{r_{v}}\left(\mathcal{O}_{v}\right)$, pour tout entier strictement positif $l$ on pose $K_{v}^{l}=1+M_{r_{v}}\left(P_{D_{v}}^{l}\right)$ et pour toute représentation lisse irréductible $\pi$ de $G L_{r_{v}}\left(D_{v}\right)$ on définit le niveau de $\pi$ comme le plus petit entier $l \geqslant 0$ tel que $\pi$ ait un vecteur fixe sous $K_{v}^{l}$.

Maintenant on applique le théorème 1.2(b) pour en déduire de (3) qu'il existe une constante $c^{\prime}$ telle que pour toute $\tilde{\pi}^{\prime} \in X$ on ait $\sum_{v \in V} \operatorname{niv}\left(\tilde{\pi}_{v}^{\prime}\right) \leqslant c^{\prime}$ (grossièrement, prendre $c^{\prime}=c+n|V|$ où $|V|$ est le cardinal de $V$ ). Comme un niveau est toujours un nombre positif ou nul, pour toute $\tilde{\pi}^{\prime} \in X$ on a

$$
\forall v \in V, \quad \operatorname{niv}\left(\tilde{\pi}_{v}^{\prime}\right) \leqslant c^{\prime} .
$$

Pour toute place finie $v$ de $F$ on définit un entier $l_{v}$ de la façon suivante: si $v \in V$, $l_{v}=c^{\prime}$ et si $v \notin V, l_{v}=\operatorname{niv}\left(\pi_{v}\right)$. Pour presque toute place finie $v$ on a $\operatorname{niv}\left(\pi_{v}\right)=0$, sinon 
$X$ serait vide (on sait qu'une représentation automorphe a des composantes de niveau nul à presque toutes les places, [Fl]). Cela implique que le produit $K=\prod K_{v}^{l_{v}}$ est un sous-groupe ouvert compact de la partie finie du groupe $A^{*}(\mathrm{~A})$. Si la caractéristique de $F$ est non nulle et il n'y pas de palaces infinies, de l'inégalité (4) il résulte que, pour toute classe (automorphe cuspidale irréductible) dans $X$, tout représentant admet un vecteur non nul fixe sous $K$. Par la prop.5.2 de [BJ], ces représentations sont des composantes d'une sous-représentation de dimension finie de la représentation par translation à droite dans l'espace des formes cuspidales (car le caractère central global est fixé). Il existe donc seulement un nombre fini de telles classes et le résultat est prouvé. Si la caractéristique de $F$ est nulle et il existe des places infinies, alors on a un argument analogue, en utilisant cette fois le 4.3(i) de [BJ], sachant que la partie infinie des éléments de $\mathrm{X}$ est fixée.

\section{Preuve du théorème 1.2(a)}

Nous reprenons les notations de la section 2. Dans cette section 4, la caractéristique de $F$ sera supposée nulle. Cette condition sur la caractéristique peut être évitée en montrant que $[\mathrm{BF}]$ fonctionne en toute caractéristique et donc la preuve qui suit s'applique directement au cas de caractéristique non nulle. Nous ne l'avons pas fait ici par commodité. En effet, dans un article futur nous sommes amenés à montrer qu'en se donnant une représentation cuspidale de $G L_{r}(D)$ où le corps de base $F$ est de caractéristique non nulle, on peut trouver toujours un corps local $L$ de caractéristique nulle, une algèbre à division $D_{L}$ centrale sur $L$ et une représentation cuspidale $\pi_{L}$ de $G L_{r}\left(D_{L}\right)$ tels que $\operatorname{dim}_{L} D_{L}=\operatorname{dim}_{F} D, \operatorname{niv}\left(\pi_{L}\right)=\operatorname{niv}(\pi)$ et $m\left(\pi_{L}\right)=m(\pi)$. Un corollaire immédiat sera la valabilité de 1.2(a) en toute caractéristique (et par conséquent de tous les résultats de l'article présent).

On fixe un caractère additif $\psi$ de $F$ de la façon suivante: si $F$ est une extension de $\mathbb{Q}_{p}$ alors $\psi$ est donné par la composition de la trace réduite de $F$ sur $\mathbb{Q}_{p}$ avec les applications évidentes

$$
\mathbb{Q}_{p} \rightarrow \mathbb{Q}_{p} / \mathbb{Z}_{p} \simeq \mathbb{Z}[1 / p] / \mathbb{Z} \subset \mathbb{R} / \mathbb{Z}
$$

après avoir identifié, par l'application $z \mapsto \exp (2 i \pi z), \mathbb{R} / \mathbb{Z}$ avec le groupe multiplicatif des éléments de module 1 dans $\mathbb{C}$.

Afin de montrer le théorème 1.2(a), nous allons utiliser le résultat de Bushnell et Fröhlich énoncé plus bas. Mais d'abord, nous avons besoin de quelques définitions:

Soient $t$ et $t^{\prime}$ deux entiers positifs tels que $t t^{\prime}=r$. Soit $\mathfrak{V}_{t, t^{\prime}}$ le sous-anneau de $A$ formé des matrices par blocs $\left(A_{i j}\right)_{1 \leqslant i, j \leqslant t^{\prime}}$ telles que $A_{i j} \in M_{t}\left(\mathcal{O}_{D}\right)$ si $i \geqslant j$ et $A_{i j} \in M_{t}\left(P_{D}\right)$ si $i<j$. Un tel anneau $\mathfrak{H}_{t, t^{\prime}}$ est appelé ordre principal standard de $A$. On appelle ordre principal de $A$ un sous-anneau de $A$ qui est conjugué par un élément de $G$ à un ordre principal standard (voir 1.2.15 et 1.5.2 de [BF]). Si $\mathfrak{A}_{t, t^{\prime}}$ est un ordre principal standard de $A$, alors son radical de Jacobson $\mathfrak{B}_{\mathfrak{Q}}$ est formé des matrices par blocs $\left(A_{i j}\right)_{1 \leqslant i, j \leqslant t^{\prime}}$ où $A_{i j} \in M_{t}\left(\mathcal{O}_{D}\right)$ si $i>j$ et $A_{i j} \in M_{t}\left(P_{D}\right)$ si $i \leqslant j$. On définit alors les groupes suivants: $U_{0}(\mathfrak{U})=\mathfrak{H}^{*}$ et, pour $j \geqslant 1, \quad U_{j}(\mathfrak{Q})=1+\mathfrak{P}_{\mathfrak{A}}^{j}$. L'ensemble 
$\left\{U_{j}(\mathfrak{Q})\right\}_{j \in \mathbb{N}}$ est une base de voisinages (ouverts et compacts) de l'unité dans $G$. Si on fixe un ordre principal standard $\mathfrak{H}$, si $\rho$ est une représentation d'un sous-groupe de $G$ contenant $U_{0}(\mathfrak{R})$, on pose $f(\rho)_{\mathfrak{Q}}=\mathfrak{P}_{\mathfrak{A}}^{j}$ où $j$ est le plus petit entier tel que $U_{j}(\mathfrak{Q}) \subset \operatorname{ker}(\rho)$. On appelle $f(\rho)_{\mathfrak{Q}}$ le conducteur de $\rho$ relatif à $\mathfrak{A}$. Pour la définition d'une représentation non dégénérée au sens de Bushnell-Fröhlich le lecteur est prié de se reporter à l'appendice du présent article. Elle se trouve aussi à la page 228 de $[\mathrm{BF}]$.

Si $\mathfrak{A}$ est un ordre principal de $A$, on pose

$$
\mathfrak{D}_{\mathfrak{H}}=\left\{x \in A: \psi\left(\operatorname{Tr} d_{A / F}(x y)\right)=1 \quad \forall y \in \mathfrak{Q}\right\},
$$

où $\operatorname{Tr} d$ est la trace réduite. Avec ces notations on a le résultat suivant, qui est le théorème 3.3.8(iv) dans [BF]:

PROPOSITION 4.1. Soit $\pi$ une représentation lisse irréductible cuspidale de G. Soit $\mathfrak{U}$ un ordre principal standard et $N(\mathfrak{U})$ le normalisateur de $\mathfrak{A}$ dans $G$. Supposons que la restriction de $\pi$ à $N(\mathfrak{V})$ contienne une représentation non dégénérée $\rho$ de $N(\mathfrak{Q})$. Alors le facteur $\epsilon$ de $\pi$ est donné par:

$$
\epsilon(\pi ; s ; \psi)=\left[\mathfrak{H}: \mathfrak{D}_{\mathfrak{H}} f(\rho)_{\mathfrak{H}}\right]^{(1 / 2-s) / n} W(\rho)
$$

où $W(\rho)$ est une constante complexe.

Nous pouvons maintenant entamer la preuve du théorème 1.2(a):

PROPOSITION 4.2. Soit $\pi$ une représentation lisse irréductible cuspidale de G. Alors on $a$

$$
\operatorname{niv}(\pi) \leqslant \frac{m(\pi)}{r}-d+2
$$

Démonstration. Nous pouvons appliquer la proposition 4.1 à $\pi$. Le fait que $\pi$ vérifie l'hypothèse de cette proposition est une conséquence immédiate de [GSZ], théorème 5.5(ii) si $\pi$ est de niveau 1 avec nos notations (niveau zéro dans le formalisme de [GSZ]), et une conséquence des résultats dans [Br], (voir appendice du présent article, th. A.2. (1)) si $\pi$ est de niveau supérieur ou égal à deux. On obtient

$$
\epsilon(\pi ; s ; \psi)=\left[\mathfrak{U}: \mathfrak{D}_{\mathscr{U}} f(\rho)_{\mathfrak{N}}\right]^{(1 / 2-s) / n} W(\rho)
$$

ou encore

$$
C(\pi) q^{(n a(\psi)-m(\pi)) s}=\left[\mathfrak{U}: \mathfrak{D}_{\mathfrak{A}} f(\rho)_{\mathfrak{Q}}\right]^{(1 / 2-s) / n} W(\rho) .
$$

Par 2.1.2(i) et 2.1.7 de [BF] on sait que $\mathfrak{D}_{\mathfrak{A}}=\mathfrak{B}_{\mathfrak{A}}^{e-1} \mathfrak{D}_{\mathcal{O}_{F}}$ où $e$ est un indice de ramification que nous ne définissons pas ici, (voir 1.4.6 et 1.4.7 de [BF]) et $\mathfrak{D}_{\mathcal{O}_{F}}$ est la différente absolue de $F$, qui, avec nos notations, n'est autre que $P_{F}^{-a(\psi)}$. Par la formule 1.4.7 de $[\mathrm{BF}]$ on a donc

$$
\mathfrak{D}_{\mathfrak{I}}=\mathfrak{P}_{\mathfrak{A}}^{(-a(\psi)) e+e-1}=\mathfrak{B}_{\mathfrak{Q}}^{(-a(\psi)+1) e-1} \text {. }
$$


Soit $j$ tel que $f(\rho)_{\mathfrak{Q}}=\mathfrak{P}_{\mathfrak{Q}}^{j}$. Nous trouvons ainsi

$$
\left[\mathfrak{U}: \mathfrak{D}_{\mathfrak{U}} f(\rho)_{\mathfrak{I}}\right]=\left[\mathfrak{U}: \mathfrak{P}_{\mathfrak{U}}^{(-a(\psi)+1) e-1+j}\right] .
$$

Par la formule 1.4.8 de [BF], nous pouvons remplacer $e$ par $d t^{\prime}$, et ensuite, en appliquant les formules 1.4.14 et 1.4.15 dans [BF], on trouve

$$
\left[\mathfrak{H}: \mathfrak{D}_{\mathfrak{H}} f(\rho)_{\mathfrak{H}}\right]=q^{n t\left((-a(\psi)+1) d t^{\prime}-1+j\right)}=q^{n^{2}(-a(\psi)+1)-n t+n t j} .
$$

On trouve donc par (5) que

$$
-n a(\psi)+m(\pi)=n(-a(\psi)+1)-t+t j
$$

et

$$
j=\frac{m(\pi)}{t}-d t^{\prime}+1
$$

soit

$$
m(\pi)-n=t(j-1)
$$

Maintenant, choisissons un vecteur $v$ non nul dans l'espace de $\rho$. Alors $v$ sera pour $\pi$ un vecteur fixe sous $\operatorname{ker}(\rho) \supseteq 1+\mathfrak{P}_{\mathfrak{q}}^{j}$. Mais, en désignant par $E(x)$ la partie entière de $x$, on a par 1.4.7 de $[\mathrm{BF}]$

$$
\mathfrak{P}_{\mathfrak{I}}^{j}=P_{D}^{E\left(\frac{j-1}{t^{\prime}}\right)} \mathfrak{P}_{\mathfrak{A}}^{j-t^{\prime} E\left(\frac{i-1}{t^{\prime}}\right)} \supset P_{D}^{E\left(\frac{j-1}{t^{\prime}}\right)} \mathfrak{P}_{\mathfrak{A}}^{t^{\prime}} \supset P_{D}^{E\left(\frac{j-1}{t^{\prime}}\right)} M_{r}\left(P_{D}^{2}\right) \supset M_{r}\left(P_{D}^{E\left(\frac{i-1}{t^{\prime}}\right)+2}\right)
$$

et par conséquent

$$
\operatorname{niv}(\pi) \leqslant E\left(\frac{j-1}{t^{\prime}}\right)+2=E\left(\frac{m(\pi)-n}{r}\right)+2 \leqslant \frac{m(\pi)-n}{r}+2 .
$$

\section{Appendice par Paul Broussous}

Cuspidales et représentations non dégénérées

\section{A.1. RAPPELS SUR LES STRATES}

Nous conservons toutes les notations de l'article (en particulier, celles du § 4.). Le corps $F$ est ici de caractéristique nulle, extension finie de $\mathbb{Q}_{p}, p$ premier.

Soit $\mathfrak{A}$ un $\mathcal{O}_{F}$-ordre héréditaire (pas forcément principal) de $A$, et soit $n$ un entier $\geqslant 1$. Alors l'application $x \mapsto x-1$ induit un isomorphisme canonique de groupes:

$$
U_{n}(\mathfrak{H}) / U_{n+1}(\mathfrak{H}) \simeq\left(\mathfrak{P}_{\mathfrak{Q}}^{n} / \mathfrak{P}_{\mathfrak{A}}^{n+1},+\right) .
$$

$\operatorname{Par}[\mathrm{BF}] \S 2$, le dual de Pontryagin de $\mathfrak{P}_{\mathfrak{A}}^{n} / \mathfrak{P}_{\mathfrak{U}}^{n+1}$ est isomorphe à $\mathfrak{D}_{\mathfrak{U}}^{-1} \mathfrak{P}_{\mathfrak{U}}^{-(n+1)} /$ $\mathfrak{D}_{\mathfrak{I}}^{-1} \mathfrak{P}_{\mathfrak{Q}}^{-n}$, via:

$\Psi: h \bmod \mathfrak{D}_{\mathfrak{I}}^{-1} \mathfrak{P}_{\mathfrak{I}}^{-n} \mapsto \psi_{h}$, 
où $\psi_{h}(u)=\psi\left(\operatorname{Tr}_{A / F}(h \tilde{u})\right), \tilde{u}$ étant un représentant de la classe $u$. Ici $\mathfrak{D}_{\mathfrak{A}}$ est la différente absolue de $\mathfrak{A}($ voir $\S 4)$; elle est donnée par $\mathfrak{D}_{\mathfrak{A}}=\mathfrak{P}_{\mathfrak{A}}^{e-1} \mathfrak{D}_{\mathcal{O}_{F}}$, où $\mathfrak{D}_{\mathcal{O}_{F}}$ est la différente de $F$ sur $\mathbb{Q}_{p}$ et $e$ l'entier défini par $P_{F} \mathfrak{U}=\mathfrak{B}_{\mathfrak{A}}^{e}$ (voir $[\mathrm{BF}]$ ). Rappelons que $\mathfrak{D}_{\mathcal{O}_{F}}=P_{F}^{\alpha}$, où $\alpha$ est l'exposant différentiel de $F / \mathbb{Q}_{p}$.

Soit $\chi$ un caractère additif lisse de $F\left(\right.$ resp. $\left.\mathbb{Q}_{p}\right)$. On définit l'ordre de $\chi, \operatorname{ord}(\chi)$, comme le plus grand entier $v$ tel que $\chi$ soit trivial sur $P_{F}^{-v}$ (resp. $p^{-v} \mathbb{Z}_{p}$ ). Le caractère $\psi$ de $F$ introduit dans la section 4. est de la forme $\psi_{\mathbb{Q}_{p}} \circ \operatorname{Tr}_{F / \mathbb{Q}_{p}}$, où $\psi_{\mathbb{Q}_{p}}$ est d'ordre 0 . Par [We], VIII, $\S 1$, cor. $3, \psi$ est d'ordre $\alpha$. On définit un second caractère additif de $F$ par $\psi^{o}(u)=\psi\left(\pi_{F}^{\alpha+1} u\right)$. Par construction, $\psi^{o}$ est d'ordre -1 ; il permet (cf. [Br]) de construire un isomorphisme entre le dual de $\mathfrak{P}_{\mathfrak{A}}^{n} / \mathfrak{P}_{\mathfrak{A}}^{n+1}$ et $\mathfrak{P}_{\mathfrak{A}}^{-n} / \mathfrak{P}_{\mathfrak{A}}^{-n+1}$ via:

$$
\Psi^{o}: k \bmod \mathfrak{P}_{\mathfrak{A}}^{-n+1} \mapsto \psi_{k}^{o},
$$

où $\psi_{k}^{o}(u)=\psi^{o}\left(\operatorname{Tr} d_{A / F}(k \tilde{u})\right)$.

Notons que

$$
\mathfrak{D}_{\mathfrak{l}}^{-1} \mathfrak{B}_{\mathfrak{l}}^{-n}=P_{F}^{-\alpha} \mathfrak{B}_{\mathfrak{l}}^{1-e} \mathfrak{B}_{\mathfrak{l}}^{-n}=P_{F}^{-\alpha} P_{F}^{-1} \mathfrak{B}^{-n+1}=P_{F}^{-\alpha-1} \mathfrak{B}_{\mathfrak{l}}^{-n+1}
$$

Il s'ensuit facilement que la multiplication par $\pi_{F}^{-(\alpha+1)}$ induit un isomorphisme de groupes:

$$
\varphi: \mathfrak{P}_{\mathfrak{l}}^{-n} / \mathfrak{P}_{\mathfrak{l}}^{-n+1} \longrightarrow \mathfrak{D}_{\mathfrak{I}}^{-1} \mathfrak{P}_{\mathfrak{I}}^{-(n+1)} / \mathfrak{D}_{\mathfrak{I}}^{-1} \mathfrak{P}_{\mathfrak{l}}^{-n} .
$$

Un calcul simple donne:

LEMME A.1.1. Le diagramme suivant est commutatif:

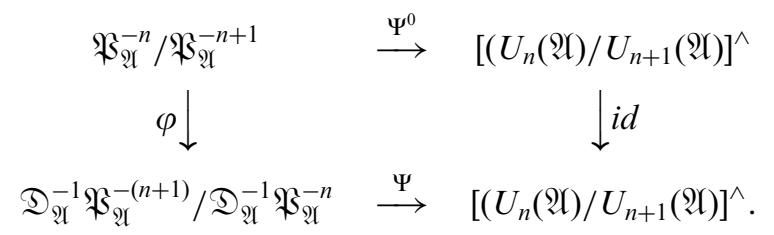

Si $\chi$ est un caractère de $U_{n}(\mathfrak{Q})$, trivial sur $U_{n+1}(\mathfrak{H})$, une strate attachée à $\chi$ (cf. [BK], [Br]) est un quadruplet $[\mathfrak{A}, n, n-1, h], h \in \mathfrak{B}_{\mathfrak{A}}^{-n}$, tel que $\Psi^{o}\left(h \bmod \mathfrak{P}_{\mathfrak{A}}^{-n+1}\right)=\chi$.

Soit $[\mathfrak{A}, n, n-1, h]$ une strate (avec, rappelons-le, $n \geqslant 1$ ). Notons $\Phi_{h}[X] \in \mathbf{k}[X]$ son polynôme caractéristique (cf. [Br] 1.1); ici $\mathbf{k}=\mathcal{O}_{F} / P_{F}$ désigne le corps résiduel. Rappelons [Br] que [ $\mathfrak{U}, n, n-1, h]$ est fondamentale si $X$ ne divise pas $\Phi_{h}[X]$, et non scindée si $\Phi_{h}[X]$ est puissance d'un polynôme irréductible. Le niveau normalisé d'une strate est par définition le rationnel $n / e$, où $e$ est l'entier défini plus haut. Par abus de langage, on dira qu'une représentation lisse $\pi$ de $G$ contient une strate $[\mathfrak{U}, n, n-1, h]$, si par restriction à $U_{n}(\mathfrak{H}), \pi$ contient le caractère $\chi$ associé.

Rappelons le 
THÉORÈME A.1.2. (Howe-Moy et Bushnell, cf.[Br] §1.) Soit $\pi$ une représentation lisse irréductible de $G$ telle que niv $(\pi) \geqslant 2$. Alors $\pi$ contient une strate fondamentale. Toutes les strates fondamentales contenues dans $\pi$ ont le même niveau normalisé. Ce nombre rationnel est noté $l(\pi)$ et s'appelle le niveau normalisé de $\pi$. De plus, si une strate de niveau $l(\pi)$ est contenue dans $\pi$, elle est fondamentale.

\section{A.2. CUSPIDALES ET REPRÉSENTATIONS NON DÉGÉNÉRÉES}

Soit $\mathfrak{A}$ un $\mathcal{O}_{F}$-ordre principal de $A$. On note toutjours $K(\mathfrak{H})$ son normalisateur dans $G$. Soit $\rho$ une représentation lisse irréductible de $K(\mathfrak{V})$, de conducteur $f(\rho)_{\mathfrak{A}}$. Supposons que $f(\rho)_{\mathfrak{A}}=\mathfrak{B}_{\mathfrak{A}}^{n+1}$, avec $n \geqslant 1$. On dit que $\rho$ est non dégénérée (cf. [BF] $\S 2$ ), si par restriction $\rho$ contient un caractère $\Psi(h)$ de $U_{n}(\mathfrak{Q})$ tel que $h+\mathfrak{D}_{\mathfrak{H}}^{-1} \mathfrak{P}_{\mathfrak{A}}^{-n} \subset K(\mathfrak{W})$. D'après le lemme (A.1.1), et puisque $\pi_{F} \in K(\mathfrak{H})$, cela revient à dire que $\rho$ contient un caractère $\Psi^{o}(h)$ tel que $h+\mathfrak{B}_{\mathfrak{A}}^{n+1} \subset K(\mathfrak{R})$. Avec un abus de langage déjà utilisé, cela revient encore à dire que $\rho$ contient une strate $[\mathfrak{H}, n, n-1, h]$ avec $h+\mathfrak{B}_{\mathfrak{A}}^{-n+1} \subset$ $K(\mathfrak{U})$.

THÉORÈME A.2.1. Soit $\pi$ une représentation lisse irréductible cuspidale de G. Supposons que $\operatorname{niv}(\pi) \geqslant 2$. Alors il existe un ordre principal $\mathfrak{A}$ et une représentation non dégénérée $\rho$ de $K(\mathfrak{H})$ telle que $\pi$ contienne $\rho$ par restriction. De plus, avec les notations précédentes, la strate $[\mathfrak{U}, n, n-1, h]$ contenue dans $\rho$ (donc dans $\pi$ ) peut être choisie de façon à avoir un niveau normalisé égal à $l(\pi)$.

La démonstation de ce théorème consiste en une série de lemmes.

Fixons $\pi$, lisse irréductible cuspidale, telle que $\operatorname{niv}(\pi) \geqslant 2$. Par (A.1.2), $\pi$ contient une strate fondamentale [ $\mathfrak{U}, n, n-1, h], n \geqslant 1$. Par [Br], Théorème (1.2.3), le fait que

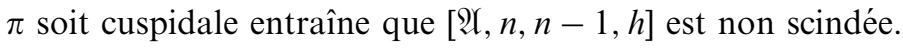

LEMME A.2.2. Si une strate $[\mathfrak{U}, n, n-1, h]$ est fondamentale non scindée, alors $h+\mathfrak{B}_{\mathfrak{A}}^{-n+1} \subset K(\mathfrak{H})$.

Démonstration. Notons $\left(L_{k}\right)_{k \in Z}$ une chaîne d' $\mathcal{O}_{D}$-réseaux (cf. [Br]) dans le $D$ espace vectoriel à droite $V=D^{m}$, dont $\mathfrak{Q}$ soit le stabilisateur dans $A$. Rappelons qu'il existe un entier $t \geqslant 1$ (la période de $\mathfrak{Q}$ ), tel que $L_{k} \pi_{D}=L_{k+t}, k \in \mathbb{Z}$. L'entier $e=t d$ est l'entier considéré plus haut. La multiplication par $h$ induit des applications $k_{D^{-}}$ linéaires $L_{k} / L_{k+1} \longrightarrow L_{k-n} / L_{k+1-n}$. Il découle facilement de la définition de $\Phi_{h}$ que ces applications linéaires sont des isomorphismes d'espaces vectoriels. Il s'ensuit que $h L_{k}=L_{k-n}$, pour tout $k$ dans $\mathbb{Z}$. Ainsi, suivant la terminologie de $[\mathrm{Br}](1.2), h$ est un automorphisme de $\left(L_{k}\right)$ et est donc contenu dans $K(\mathfrak{2})([\mathrm{BF}] \mathrm{Thm}(1.2 .8))$ ). On a $h \mathfrak{B}_{\mathfrak{A}}=\mathfrak{B}_{\mathfrak{I}}^{-n+1}$, de sorte que $h+\mathfrak{B}_{\mathfrak{A}}^{-n+1}=h\left(1+\mathfrak{B}_{\mathfrak{I}}\right)=h U_{1}(\mathfrak{K})$. On donc bien $h+\mathfrak{P}_{\mathfrak{U}}^{-n+1} \subset K(\mathfrak{H})$.

Soit $[\mathfrak{A}, n, n-1, h]$ une strate fondamentale non scindée contenue dans $\pi$. On peut toujours trouver une représentation lisse irréductible $\rho$ de $K(\mathfrak{H})$, contenue dans $\pi$ et contenant $[\mathfrak{H}, n, n-1, h]$ par restriction. Pour avoir le théorème (A.2.1), on est donc ramené á prouver le: 
LEMME A.2.3. La représentation $\pi$ contient une strate fondamentale non scindée $[\mathfrak{A}, n, n-1, h]$ avec $\mathfrak{A}$ principal.

Démonstration. Soient $[\mathfrak{H}, n, n-1, h]$ une strate fondamentale non scindée contenue dans $\pi,\left(L_{k}\right)$ une chaîne d' $\mathcal{O}_{D}$-réseaux de stabilisateur $\mathfrak{A}$ et $t$ la période de $\mathfrak{A}$. Posons $g=(n, t), n_{o}=n / g, t_{o}=t / g$. Soit $\mathcal{L}$ l'ensemble des $\mathcal{O}_{D}$-réseaux de $V$ de la forme $h^{u} L_{0} \pi_{D}^{v}, u, v \in \mathbb{Z}$. C'est l'ensemble des réseaux de la chaîne $\left(L_{k}^{o}\right)_{k \in \mathbb{Z}}$, où $L_{k}^{o}=L_{g k}$; elle est de période $t_{o}$. On a $h L_{k}^{o}=L_{k-n_{o}}^{o}$, de sorte que si $\mathfrak{H}_{o}$ est l'ordre attaché à $\left(L^{o}\right)$, on a $h \in \mathfrak{B}_{\mathfrak{R}_{o}}^{-n_{o}}$. Par construction, on peut trouver des entiers $u$ et $v$ tels que $h^{u} L_{k}^{o} \pi_{D}^{v}=L_{k+1}^{o}, k \in \mathbb{Z}$. L'application $x \mapsto h^{u} x \pi_{D}^{v}$ est un automorphisme $F$-linéaire de $V$. Il induit des automorphismes k-linéaires $L_{k}^{o} / L_{k+1}^{o} \simeq L_{k+1}^{o} / L_{k+2}^{o}$. Il s'ensuit que la suite $\operatorname{dim}_{k_{D}} L_{k}^{o} / L_{k+1}^{o}$ est constante et que l'ordre $\mathfrak{A}_{o}$ est principal ([BF] Thm (1.3.2)(iii)).

La strate $\left[\mathfrak{H}_{o}, n_{o}, n_{o}-1, h\right]$ a pour niveau normalisé $n_{o} / \mathrm{d} t_{o}=n / \mathrm{d} t=l(\pi)$. On est ramené á prouver le

LEMME A.2.4. Il existe une strate fondamentale de la forme $\left[\mathfrak{H}_{o}, n_{o}, n_{o}-1, h_{o}\right]$, contenue dans $\pi$.

Démonstration. Puisque $\left(L^{o}\right)$ contient moins de réseaux que $(L)$, on a $\mathfrak{A} \subset \mathfrak{A}_{o}$. Donc $\mathfrak{P}_{\mathfrak{Q}}^{-n}=h \mathfrak{U} \subset h \mathfrak{\mathfrak { H } _ { o }}=\mathfrak{P}_{\mathfrak{A}_{o}}^{-n_{o}}$. Via la dualité dans $A$ induite par le caractère $\psi^{o}$, on obtient $\mathfrak{P}_{\mathfrak{A}_{0}}^{n_{o}+1} \subset \mathfrak{P}_{\mathfrak{A}}^{n+1}$, c'est à dire $U_{n_{o}+1}\left(\mathfrak{H}_{o}\right) \subset U_{n+1}(\mathfrak{H})$. En particulier, $\pi$ a des vecteurs fixes sous $U_{n_{o}+1}\left(\mathfrak{H}_{o}\right)$. Regardons la restriction de $\pi$ à $U_{n_{o}}\left(\mathfrak{A}_{o}\right)$. Le $U_{n_{o}}\left(\mathfrak{H}_{o}\right)$ module engendré par les vecteurs fixes sous $U_{n_{o}+1}\left(\mathfrak{H}_{o}\right)$ fournit des strates de la forme $\left[\mathfrak{A}_{o}, n_{o}, n_{o}-1, h_{o}\right]$, contenues dans $\pi$. Elles ont pour niveau normalisé $l(\pi)$. Elles sont donc fondamentales. Ceci achève la démonstration du lemme (A.2.3) et du théoréme (A.2.1).

\section{Bibliographie}

[Be] Bernstein, J.: Le “centre" de Bernstein, rédigé par Deligne, Représentations des groupes réductifs sur un corps local, Travaux en cours, Hermann, Paris, 1984, pp.1-32.

[BF] Bushnell, C. J. et Fröhlich, A.: Non-abelian congruence sums and $p$-adic simple algebras, Proc. London Math. Soc. Ser. (3) 50 (1985), 207-264.

[BJ] Borel, A. et Jacquet, H.: Automorphic forms and automorphic representations, Proc. Sympos Pure Math. 33 (1), 189-202.

[BK] Bushnell, C. J. et Kutzko, P.: The Admissible Dual of $G L(N)$ via Compact Open Subgroups, Ann. Math. Stud. 129, Princeton Univ. Press, 1993.

[Br] Broussous, P.: Minimal strata for GL(m;D), J. reine. angew. Math 514 (1999), 199-236.

[Fl] Flath, D.: Decomposition of representations into tensor products, Proc. Sympos Pure Math. 33 (1), 179-185.

[GJ] Godement, R. et Jacquet, H.: Zeta Functions of Simple Algebras, Lecture Notes in Math 260, Springler, New York, 1972.

[GSZ] Grabitz, M., Silberger, A. and Zink, E.-W.: Level zero types and Hecke algebras for local central simple algebras, Preprint Nr.2000-6, Humbold-Universität zu Berlin. 
[Ro] Rogawski, J.: Representations of $G L(n)$ and division algebras over a $p$-adic field, Duke Math. J. 50 (1983), 161-201.

[We] Weil, A.: Basic Number Theory, Classics in Math., Springer-Verlag, Berlin, 1973. 